\title{
ON THE PERSISTENCE OF THE EIGENVALUES OF A PERTURBED FREDHOLM OPERATOR OF INDEX ZERO UNDER NONSMOOTH PERTURBATIONS
}

\author{
PIERLUIGI BENEVIERI, ALESSANDRO CALAMAI, MASSIMO FURI, \\ AND MARIA PATRIZIA PERA
}

\begin{abstract}
Let $H$ be a real Hilbert space and denote by $S$ its unit sphere. Consider the nonlinear eigenvalue problem $L x+\varepsilon N(x)=\lambda x$, where $\varepsilon, \lambda \in \mathbb{R}, L: H \rightarrow H$ is a bounded self-adjoint (linear) operator with nontrivial kernel and closed image, and $N: H \rightarrow H$ is a (possibly) nonlinear perturbation term. A unit eigenvector $\bar{x} \in S \cap \operatorname{Ker} L$ of $L$ (corresponding to the eigenvalue $\lambda=0$ ) is said to be persistent if it is close to solutions $x \in S$ of the above equation for small values of the parameters $\varepsilon \neq 0$ and $\lambda$. We give an affirmative answer to a conjecture formulated by R. Chiappinelli and the last two authors in an article published in 2008. Namely, we prove that if $N$ is Lipschitz continuous and the eigenvalue $\lambda=0$ has odd multiplicity, then the sphere $S \cap \operatorname{Ker} L$ contains at least one persistent eigenvector. We provide examples in which our results apply, as well as examples showing that if the dimension of Ker $L$ is even, then the persistence phenomenon may not occur.
\end{abstract}

\section{INTRODUCTION}

Let $L$ be a self-adjoint operator defined on a real Hilbert space $H$. Assume that $\lambda_{0} \in \mathbb{R}$ is an isolated eigenvalue of $L$ and let $N: H \rightarrow H$ be a Lipschitz continuous map. Consider the problem

$$
\left\{\begin{array}{l}
L x+\varepsilon N(x)=\lambda x \\
\|x\|=1
\end{array}\right.
$$

where $\varepsilon$ and $\lambda$ are real parameters. Assume, for the moment, that the eigenvalue $\lambda_{0}$ is simple. Thus, when $\varepsilon=0$ and $\lambda=\lambda_{0}$, one gets exactly two vectors in $H$ satisfying the above problem; namely, the two unit eigenvectors of $L$ corresponding to the eigenvalue $\lambda_{0}$. Denote by $x^{1}$ and $x^{2}$ these vectors. Considering small values of $\varepsilon, \mathrm{R}$. Chiappinelli in [11] obtained a sort of persistence result of these eigenvectors as well as of the eigenvalue $\lambda_{0}$. More

Date: January 18, 2021.

2010 Mathematics Subject Classification. Primary 47J10; Secondary 47A75, 35P30.

Key words and phrases. Fredholm operators, nonlinear spectral theory, eigenvalues, eigenvectors, degree theory.

A. Calamai is partially supported by G.N.A.M.P.A. - INdAM (Italy).

The first, second and fourth authors are members of the Gruppo Nazionale per l'Analisi Matematica, la Probabilità e le loro Applicazioni (GNAMPA) of the Istituto Nazionale di Alta Matematica (INdAM). 
precisely, he proved that, defined in a neighborhood $V$ of $0 \in \mathbb{R}$, there exist two $H$-valued Lipschitz curves, $\varepsilon \mapsto x_{\varepsilon}^{1}$ and $\varepsilon \mapsto x_{\varepsilon}^{2}$, as well as two real Lipschitz functions, $\varepsilon \mapsto \lambda_{\varepsilon}^{1}$ and $\varepsilon \mapsto \lambda_{\varepsilon}^{2}$, such that for $i=1,2$ and $\varepsilon \in V$ one has

$$
L x_{\varepsilon}^{i}+\varepsilon N\left(x_{\varepsilon}^{i}\right)=\lambda^{i}(\varepsilon) x_{\varepsilon}^{i}, \quad\left\|x_{\varepsilon}^{i}\right\|=1 .
$$

In particular, when $\varepsilon=0$, these four functions satisfy the following conditions: $x_{0}^{i}=x^{i}, \lambda_{0}^{i}=\lambda_{0}$.

After the result of Chiappinelli, a natural question emerged: what can one say if the multiplicity of the eigenvalue $\lambda_{0}$ is bigger than one? In this case the set of unit eigenvectors of $L$ corresponding to $\lambda_{0}$ is the $(n-1)$ dimensional unit sphere $S^{n-1}$ of $\operatorname{Ker}\left(L-\lambda_{0} I\right)$, where $I$ is the identity of $H$ and $n$ is the multiplicity of $\lambda_{0}$.

We may informally ask two questions:

- Given an element $\bar{x} \in S^{n-1}$, under what necessary, as well as sufficient, conditions the persistence of $\bar{x}$ occurs?

- Does the persistence of some unit eigenvector still subsist when $n>1$ and $\varepsilon$ is small?

In order to make the expression "persistence of an unit eigenvector" more precise, we introduce some terminology.

Denote by $S$ the unit sphere in $H$. By a solution of (1.1) we mean a triple $(x, \varepsilon, \lambda) \in S \times \mathbb{R} \times \mathbb{R}$ verifying the equation $L x+\varepsilon N(x)=\lambda x$. In the set of all solutions of (1.1) we distinguish the subset $S^{n-1} \times\{0\} \times\left\{\lambda_{0}\right\}$ of the trivial ones, so that the notion of nontrivial solution is well defined. We say that an element of $\bar{x} \in S^{n-1}=S \cap \operatorname{Ker}\left(L-\lambda_{0} I\right)$ is a bifurcation point (or a persistent eigenvector) for problem (1.1) if any neighborhood of the associated trivial solution $\left(\bar{x}, 0, \lambda_{0}\right)$ contains nontrivial solutions.

Regarding the first question, in [12] a necessary condition for $\bar{x}$ to be a bifurcation point was given, but under the restrictive assumption that the perturbing operator $N$ is of class $C^{1}$ (in a neighborhood of $\bar{x}$ ). Moreover, a sufficient condition was obtained, provided that $N$ is $C^{2}$ (near $\bar{x}$ ). In the same paper the authors conjectured that, if the multiplicity $n$ of $\lambda_{0}$ is odd, then some unit eigenvector is a bifurcation point. Elementary examples show that if $n$ is even, then the persistence phenomenon (i.e. the existence of a bifurcation point) may not occur, unless $N$ is a gradient operator (as proved in [14]).

A first positive, but not complete, answer to the above conjecture was given in [13]. It was shown that the persistence phenomenon occurs if the operator $N$ is assumed to be $C^{1}$. The proof is based on the Lefschetz Fixed Point Theorem, taking into account that, if $n$ is odd, then the EulerPoincaré characteristic of $S^{n-1}$ is different from zero (it is actually 2).

In [15] the persistence problem was investigated in the more general context of real Banach spaces. Namely, it was considered the system

$$
\left\{\begin{array}{l}
L x+\varepsilon N(x)=\lambda C x \\
g(x)=1
\end{array}\right.
$$


where $L, C: E \rightarrow F$ are bounded linear operators between real Banach spaces, $N: E \rightarrow F$ is a nonlinear map, and $g$ is a continuous norm in $E$, not necessarily equivalent to the Banach norm of $E$. Under a natural transversality condition between $L$ and $C$ (see (5.2) or (5.3) below), it was shown that if $L$ is Fredholm of index zero with odd dimensional kernel and $N$ is $C^{1}$, then one obtains the persistence of at least one element of $g^{-1}(0) \cap \operatorname{Ker} L$. Notice that, in problem (1.1), without loss of generality one may assume $\lambda_{0}=0$. Therefore, this new problem includes the previous one.

The persistence result in [15], however, does not include the most frequent case in which $N$ is a completely continuous map, nor the case in which $N$ is Lipschitz continuous, as conjectured in [12]. The purpose of this paper is to fill this gap, allowing $N$ to be locally $\alpha$-Lipschitz, where $\alpha$ stands for the Kuratowski measure of noncompactness. Obviously, this includes the case in which $N$ is the sum of a locally Lipschitz (e.g. $C^{1}$ ) map and a completely continuous nonlinear operator.

In other words, here we give a positive complete answer to the conjecture formulated in [12]. What is more, instead of merely considering (as candidates for persistence) eigenvectors of the type $g^{-1}(0) \cap \operatorname{Ker} L$, we seek for vectors belonging to $\Sigma:=\partial \Omega \cap \operatorname{Ker} L$, where $\Omega$ is any open set of $E$ containing the origin and such that $\Sigma$ is nonempty and compact, as it is $g^{-1}(0) \cap \operatorname{Ker} L$, due to the fact that $g$ is a norm and $\operatorname{Ker} L$ is nontrivial and finite dimensional.

The results we obtain here are based upon a notion of degree, developed in $[2,3]$, for a class of noncompact perturbations of Fredholm maps of index zero between Banach spaces, called $\alpha$-Fredholm maps, whose definition is related to the measure of noncompactness $\alpha$. For the reader's convenience, in Sections 3 and 4 we recall and summarize the main points of the construction of the degree for $\alpha$-Fredholm maps (or, more precisely, for $\alpha$-Fredholm triples).

We conclude the paper providing examples in which our results apply, as well as examples showing that the assumption that the dimension of Ker $L$ is odd cannot be dropped.

Acknowledgment. One of the referees of this paper pointed out to us that some particular cases of our persistence result are strongly related to the Rabinowitz global bifurcation theorem [24] and its generalizations to one parameter families of maps (as in [23] and [7]). We appreciate the witty argument exposed by the referee for the case of problem (1.2) with $N$ locally Lipschitz and mapping the, possibly unbounded, unit $g$-sphere into a bounded set. We thank her/him for having made us aware of the close interesting relation.

\section{Preliminaries}

Let $f: X \rightarrow Y$ be a continuous map between two metric spaces. We recall that $f$ is said to be compact if $f(X)$ is relatively compact, and completely 
continuous if it is compact on any bounded subset of $X$. If for any $p \in X$ there exists a neighborhood $U$ of $p$ such that the restriction $\left.f\right|_{U}$ is compact, then $f$ is called locally compact. The map $f$ is said to be proper if $f^{-1}(\mathcal{K})$ is compact for any compact subset $\mathcal{K}$ of $Y$ and locally proper if for any $p \in X$ there exists a closed neighborhood $U$ of $p$ such that the restriction $\left.f\right|_{U}$ is proper. Recall that a proper map sends closed sets into closed sets.

A multivalued map $\phi: X \multimap Y$ between two metric spaces is said to be upper semicontinuous if it has compact (possibly empty) values and for any open subset $V$ of $Y$ the upper inverse image of $V$, i.e. the set $\phi^{-1}(V)=\{x \in$ $X: \phi(x) \subseteq V\}$, is an open subset of $X$. Clearly, the composition of upper semicontinuous maps is upper semicontinuous.

The following elementary remark will be used in the sequel.

Remark 2.1. Let $\mathcal{K}$ be a compact subset of $X \times Y$ and, for any $x \in X$, denote by $\mathcal{K}_{x}=\{y \in Y:(x, y) \in \mathcal{K}\}$ the slice of $\mathcal{K}$ at $x$. Then, the multivalued map $x \in X \mapsto \mathcal{K}_{x}$ (whose graph is $\mathcal{K}$ ) is upper semicontinuous. To see this, let $V$ be any open subset of $Y$ and assume, by contradiction, that the set $U=\left\{x \in X: \mathcal{K}_{x} \subseteq V\right\}$ is not open. Then, there exists a sequence $\left\{x_{n}\right\}$ in $X \backslash U$ which converges to some $x_{0} \in U$. For any $n \in \mathbb{N}$, choose $y_{n} \in \mathcal{K}_{x_{n}} \cap(Y \backslash V)$. Because of the compactness of $\mathcal{K}$, we may assume $\left(x_{n}, y_{n}\right) \rightarrow\left(x_{0}, y_{0}\right) \in \mathcal{K}$. Thus, $y_{0}$ belongs to $\mathcal{K}_{x_{0}}$ which is a subset of $V$, contradicting the fact that $y_{0}$ also belongs to the closed set $Y \backslash V$.

Let $E$ and $F$ be two real Banach spaces and let $L(E, F)$ denote the Banach space of all bounded linear operators from $E$ into $F$.

As is traditionally used in the literature, the locally compact operators of $L(E, F)$ will be simply called compact operators. Notice that they are actually completely continuous.

Recall that an operator $L \in L(E, F)$ is called semi-Fredholm if its image, $\operatorname{Im} L$, is closed and at least one of the vector spaces $\operatorname{Ker} L$ and $\operatorname{coKer} L:=$ $F / \operatorname{Im} L$ has finite dimension. Precisely, $L$ is left semi-Fredholm in the first case and right semi-Fredholm in the second one (notice that when coKer $L$ is finite dimensional, the image of $L$ is necessarily closed). The index of $L$ is the extended integer

$$
\text { ind } L=\operatorname{dim} \operatorname{Ker} L-\operatorname{dim} \operatorname{coKer} L \text {. }
$$

That is, it is either an integer or plus or minus infinity. A semi-Fredholm operator is simply called Fredholm when its index is finite. For example, one can verify that any linear operator from $\mathbb{R}^{k}$ to $\mathbb{R}^{s}$ is Fredholm of index $k-s$.

For short, a semi-Fredholm operator of index $r \in \overline{\mathbb{Z}}=\{-\infty\} \cup \mathbb{Z} \cup\{+\infty\}$ will be called a $\bar{\Phi}_{r}$-operator, or a $\bar{\Phi}$-operator if its index is not specified. The set of semi-Fredholm operators of index $r$ from $E$ into $F$ will be denoted by $\bar{\Phi}_{r}(E, F)$, while $\bar{\Phi}(E, F)$ is the set of all semi-Fredholm operators. The Greek letter $\Phi$ (instead of $\bar{\Phi}$ ) will be used to denote the special case of Fredholm operators. 
It is known that, given any $r \in \overline{\mathbb{Z}}, \bar{\Phi}_{r}(E, F)$ is an open subset of $L(E, F)$; therefore, so are the sets $\bar{\Phi}(E, F)$ and $\Phi(E, F)$. Moreover, if $L \in \bar{\Phi}_{r}(E, F)$ and $T \in L(E, F)$ is a compact operator, then $L+T \in \bar{\Phi}_{r}(E, F)$.

The following important property of Fredholm operators will be utilized in Section 6:

The composition of two Fredholm operators is a Fredholm operator whose index is the sum of the indices of the composite operators.

Actually, the same assertion holds true for the semi-Fredholm case, provided it makes sense in $\overline{\mathbb{Z}}$ the sum of the two indices.

For more information about the properties of semi-Fredholm and Fredholm operators we suggest [21] and [26].

\section{ORIEnTATION AND DEGREE FOR QUASI-FREDHOLM MAPS}

In this section we summarize the notions of orientability and degree for the quasi-Fredholm maps, introduced in [8]. The starting point is a concept of orientation for Fredholm linear operators of index zero between real Banach spaces.

Given $L \in \Phi_{0}(E, F)$, a bounded linear operator $A: E \rightarrow F$ with finite dimensional image is called a corrector of $L$ if $L+A$ is invertible. Notice that the set of correctors of $L$ is nonempty. This is true, and of crucial importance in what follows, even when $L$ does not need to be corrected (i.e. when it is invertible). On the set $\mathcal{C}(L)$ of correctors of $L$ one has an equivalence relation as follows. Let $A, B \in \mathcal{C}(L)$ be given and consider the following automorphism of $E$ :

$$
T=(L+B)^{-1}(L+A)=I-(L+B)^{-1}(B-A),
$$

where $I$ denotes the identity in $E$. The operator $K=I-T=(L+$ $B)^{-1}(B-A)$ has clearly finite dimensional image. Hence, given any finite dimensional subspace $E_{0}$ of $E$ containing $K(E)$, the restriction of $T$ to $E_{0}$ is an automorphism. Consequently, its determinant is well defined and nonzero (it is 1 when $E_{0}$ is the trivial subspace $\{0\}$ of $E$, and this occurs only in the case when $T$ is the identity). It is easy to check that this number does not depend on the choice of $E_{0}$. Thus, it makes sense to define the determinant of $T$ as the determinant of the restriction of $T$ to any finite dimensional subspace of $E$ containing the image of $K$. One says that $A$ is equivalent to $B$ or, more precisely, $A$ is $L$-equivalent to $B$, if

$$
\operatorname{det}\left((L+B)^{-1}(L+A)\right)>0 .
$$

As shown in [5], this is an equivalence relation on $\mathcal{C}(L)$ with two equivalence classes. Let us now recall the definitions of algebraic orientation of a $\Phi_{0}$-operator and of natural orientation of an isomorphism.

Definition 3.1. Let $L$ be a linear Fredholm operator of index zero between two real Banach spaces. Each one of the two equivalence classes of $\mathcal{C}(L)$ is called an orientation of $L$, and $L$ is oriented when an orientation is chosen. 
Given an oriented operator $L$, we call positive correctors of $L$ the elements of its orientation.

Definition 3.2. An oriented isomorphism $L$ is said to be naturally oriented if the trivial (i.e. null) operator is a positive corrector, and its orientation is called the natural orientation of $L$.

Definition 3.3. Let $L \in \Phi_{0}(E, F)$ be oriented. Its sign is the integer

$$
\operatorname{sign} L=\left\{\begin{aligned}
+1 & \text { if } L \text { is invertible and naturally oriented, } \\
-1 & \text { if } L \text { is invertible and not naturally oriented, } \\
0 & \text { if } L \text { is not invertible. }
\end{aligned}\right.
$$

An orientation of a Fredholm operator of index zero induces an orientation to any sufficiently close operator. Precisely, consider a Fredholm operator of index zero $L$ and a corrector $A$ of $L$. Since the set of the isomorphisms from $E$ into $F$ is open in the space $L(E, F)$ of bounded linear operators, $A$ turns out to be a corrector of every $T$ in a suitable neighborhood $W$ of $L$ in $L(E, F)$. Therefore, if $L$ is oriented and $A$ is a positive corrector of $L$, any $T \in W$ can be oriented regarding $A$ as a positive corrector of $T$. This allows to introduce the notion of topological orientation of a $\Phi_{0}(E, F)$-valued map.

Definition 3.4. Let $X$ be a topological space and $h: X \rightarrow \Phi_{0}(E, F)$ a continuous map. An orientation of $h$ is a continuous choice of an orientation $\beta(x)$ of $h(x)$ for each $x \in X$, where 'continuous' means that for any $x \in X$ there exists $A \in \beta(x)$ which is a positive corrector of $h(u)$ for any $u$ in a neighborhood of $x$. The map $h$ is orientable when it admits an orientation and oriented when an orientation is chosen.

Let us now recall the notion of orientability for Fredholm maps of index zero between real Banach spaces, given in $[5,6]$.

Recall that, given an open subset $\Omega$ of $E$, a map $g: \Omega \rightarrow F$ is a Fredholm map if it is $C^{1}$ and its Fréchet derivative, $g^{\prime}(x)$, is a Fredholm operator for all $x \in \Omega$. The index of $g$ at $x$ is the index of $g^{\prime}(x)$ and $g$ is said to be of index $n$ if it is of index $n$ at any point of its domain.

Hereafter, a nonlinear Fredholm map of index zero will be also called a $\Phi_{0}$-map. Notice that a $\Phi_{0}$-operator $L: E \rightarrow F$ is also a $\Phi_{0}$-map, being differentiable at any $x \in E$ with $L^{\prime}(x)=L$. According to a result of S. Smale (see [25]), a Fredholm map defined on an open subset of a Banach space is locally proper.

Definition 3.5 (Topological orientation of a $\Phi_{0}$-map). An orientation of a Fredholm map of index zero $g: \Omega \rightarrow F$ is an orientation of the derivative $g^{\prime}: \Omega \rightarrow \Phi_{0}(E, F)$, in the sense of Definition 3.4. Moreover, $g$ is orientable, or oriented, if so is $g^{\prime}$.

We point out that if $L: E \rightarrow F$ is a $\Phi_{0}$-operator, then it is orientable if regarded as a $\Phi_{0}$-map; that is, in the sense of Definition 3.5. In fact, at any $x \in E$, the derivative $L^{\prime}(x)$ coincides with $L$, which can be "constantly" 
oriented by choosing one of the two orientations according to Definition 3.1 (the algebraic one). To better understand the difference between the algebraic and the topological orientations, think about the restriction of $L$ to an open subset $U$ of $E$. Of course $\left.L\right|_{U}$ is orientable, and as a $\Phi_{0}$-map it admits $2^{r}$ different orientations (based on Definition 3.5), $r$ being the cardinality of the connected components of $U$. Nevertheless, only two of these orientations could be inherited from an algebraic one.

Hereafter, unless otherwise stated, an algebraically oriented Fredholm operator of index zero, as well as its restriction to any open set, will be tacitly assumed to be topologically oriented according to the orientation inherited from the assigned algebraic one.

Let $U$ be open in $E$ and let $H: U \times[0,1] \rightarrow F$ be a continuous map. We say that $H$ is a homotopy of Fredholm maps of index zero or, simply, a $\Phi_{0}$-homotopy if it is continuously differentiable with respect to the first variable and any partial map $H_{s}:=H(\cdot, s)$ is a $\Phi_{0}$-map.

Definition 3.6 (Topological orientation of a $\Phi_{0}$-homotopy). Let $H: U \times$ $[0,1] \rightarrow F$ be a $\Phi_{0}$-homotopy. An orientation of $H$ is an orientation of the partial derivative

$$
\partial_{1} H: U \times[0,1] \rightarrow \Phi_{0}(E, F), \quad(x, s) \mapsto\left(H_{s}\right)^{\prime}(x),
$$

according to Definition 3.4. Moreover, $H$ is orientable, or oriented, if so is $\partial_{1} H$.

The following result regards an important property of the notion of orientability. Roughly speaking, it is a sort of continuous transport of an orientation along a homotopy (see [6, Theorem 3.14]).

Theorem 3.7 (Orientation transport). Let $H: U \times[0,1] \rightarrow F$ be a $\Phi_{0^{-}}$ homotopy. Given any $s \in[0,1]$, assume that the partial map $H_{s}$ is oriented. Then there exists and is unique an orientation of $H$ which is compatible with that of $H_{s}$.

Way of saying. According to Theorem 3.7, if $H: U \times[0,1] \rightarrow F$ is a $\Phi_{0}$-homotopy, an orientation of $H_{0}$ induces an orientation to $H_{1}$ through the unique orientation of $H$ which is compatible with that of $H_{0}$. In this case we say that the orientations of $H_{0}$ and $H_{1}$ are linked (one to the other) through $H$.

We are now ready to recall the concepts of orientability and degree for quasi-Fredholm maps, introduced in [8].

Definition 3.8. Let $\Omega$ be an open subset of $E, g: \Omega \rightarrow F$ a Fredholm map of index zero and $k: \Omega \rightarrow F$ a locally compact map. The map $f: \Omega \rightarrow F$, defined by $f=g-k$, is called a quasi-Fredholm map and $g$ is a smoothing map of $f$.

Definition 3.9. A quasi-Fredholm map $f: \Omega \rightarrow F$ is orientable if it has an orientable smoothing map. If $f$ is orientable, an orientation of $f$ is given by choosing an oriented smoothing map. 
The above definition is well posed because, as shown in [8], if $f$ is an orientable quasi-Fredholm map, the following facts hold:

i) any smoothing map of $f$ is orientable;

ii) an orientation of a smoothing map $f$ determines uniquely an orientation of any other smoothing map.

If $f$ is oriented and $g$ is an oriented smoothing map that determines the orientation of $f$, we will refer to $g$ as a positively oriented smoothing map of $f$.

Definition 3.10. Let $f: \Omega \rightarrow F$ be an oriented quasi-Fredholm map and $U$ an open subset of $\Omega$. The triple $(f, U, 0)$ is said to be $q F$-admissible provided that $f^{-1}(0) \cap U$ is compact.

The degree for quasi-Fredholm maps is an integer valued map, defined in the set of the $\mathrm{qF}$-admissible triples and denoted $\operatorname{deg}_{\mathrm{qF}}(f, U, 0)$. The definition of degree is based on the notion of Brouwer degree by means of a suitable finite-dimensional reduction.

The degree for quasi-Fredholm maps verifies classical properties in degree theory, as additivity and homotopy invariance. The reader can find details in $[8]$.

An additional property that we will need later is the following one (see $[4]):$

(Sign) Let $L \in L(E, F)$ be an oriented isomorphism. Then

$$
\operatorname{deg}_{\mathrm{qF}}(L, E, 0)=\operatorname{sign}(L)
$$

\section{Degree For $\alpha$-Fredholm triples}

Here we summarize the notion of degree for $\alpha$-Fredholm triples given in [2]; hereafter called $\alpha$-Fredholm degree (compare also with [27]). We start by recalling the definition of the Kuratowski measure of noncompactness together with some related concepts. For general references see e.g. [18] or $[22]$.

Let $A$ be a bounded subset of a metric space $X$. The Kuratowski measure of noncompactness $\alpha(A)$ of $A$ is defined as the infimum of the real numbers $d>0$ such that $A$ admits a finite covering by sets of diameter less than or equal to $d$.

Proposition 4.1 below summarizes the main properties of the measure of noncompactness. Given a Banach space $E$, a subset $A$ of $E$ and a subset $\mathcal{C}$ of $\mathbb{R}$, we denote by $\mathcal{C} A$ the set $\{c x: c \in \mathcal{C}, x \in A\}$ and by $\overline{\operatorname{co}} A$ the closed convex hull of $A$. 
Proposition 4.1. Given two bounded subsets $A$ and $B$ of $E$, we have

$$
\begin{aligned}
& \alpha(A)=0 \text { if and only if } A \text { is totally bounded; } \\
& \text { if } A \subseteq B, \text { then } \alpha(A) \leq \alpha(B) ; \\
& \alpha(A \cup B)=\max \{\alpha(A), \alpha(B)\} \\
& \alpha(A+B) \leq \alpha(A)+\alpha(B) \\
& \alpha(\mathcal{C} A)=\sup \{|c|: c \in \mathcal{C}\} \alpha(A) \\
& \alpha(\overline{\mathrm{cO}} A)=\alpha(A) .
\end{aligned}
$$

Properties (4.1)-(4.5) are easy consequences of the definition, while the last one is due to Darbo [17]. Obviously, the first three properties hold true also for subsets of a metric space.

Let $f: X \rightarrow Y$ be a map between metric spaces. In [20] (see also [1]) the map $f$ is called $\alpha$-Lipschitz of constant $k \geq 0$ if $\alpha(f(A)) \leq k \alpha(A)$ for any bounded subset $A \subseteq X$. Since in [20] the measure on noncompactness $\alpha$ is considered only for bounded sets, in the inequality $\alpha(f(A)) \leq k \alpha(A)$ it is tacitly assumed that $f(A)$ is bounded. Thus, an $\alpha$-Lipschitz map sends bounded sets into bounded sets. For short, a map that satisfies this last property will be called $\alpha$-admissible.

We say that an $\alpha$-admissible map $f$ is $\alpha$-coercive if there exists $c>0$ (called an $\alpha$-coercive constant of $f$ ) such that $\alpha(f(A)) \geq c \alpha(A)$ for any bounded $A \subseteq X$.

Given an $\alpha$-admissible map $f: X \rightarrow Y$, in [20] the following two nonnegative extended real numbers are defined:

$$
\begin{aligned}
& \alpha(f)=\inf \{k \geq 0: \alpha(f(A)) \leq k \alpha(A), \text { for any bounded } A \subseteq X\}, \\
& \omega(f)=\sup \{c \geq 0: \alpha(f(A)) \geq c \alpha(A), \text { for any bounded } A \subseteq X\} .
\end{aligned}
$$

Observe that each of these two numbers can be $+\infty$. The first one when (and only when) $f$ is not $\alpha$-Lipschitz, according to the convention that the infimum of the empty set is $+\infty$ (in fact, because of the vacuous truth, any real number is a lower bound of $\emptyset$ ). The second one, for example, when any bounded subset of the domain $X$ of the ( $\alpha$-admissible) map $f$ is totally bounded.

Given any bounded subset $A$ of $X$, from the above definitions one gets the inequalities

$$
\alpha(f(A)) \leq \alpha(f) \alpha(A) \text { and } \omega(f(A)) \geq \omega(f) \alpha(A),
$$

whenever they make sense (that is, except in the case when $\alpha(A)=0$ and either $\alpha(f)$ or $\omega(f)$ is $+\infty)$.

Remark 4.2. Let $f: X \rightarrow Y$ be continuous and $\alpha$-admissible. Clearly, $\alpha(f)=0$ if and only if $f$ maps bounded sets into precompact set (i.e. totally bounded). Therefore, if the metric space $Y$ is complete, then $\alpha(f)=0$ means that $f$ is completely continuous. Regarding the extended real number $\omega(f)$, we point out that, if it is positive (that is, if $f$ is $\alpha$-coercive) and $X$ is complete, then $f$ is proper on any bounded closed subset of $X$. The converse 
is not true (think about the homeomorphism $x \mapsto\|x\| x$ defined in an infinite dimensional Banach space).

We point out that $\omega(f)$ in [20] is denoted by $\beta(f)$; however, as in [19] we prefer the notation $\omega(f)$, which is motivated by the fact that $\omega$ is the last letter of the Greek alphabet.

For future reference we recall some properties of $\alpha(f)$ and $\omega(f)$. See [20] for a comprehensive list and for the proofs.

Proposition 4.3. Let $f$ and $g$ be two $\alpha$-admissible maps from a metric space $X$ into a Banach space $F$. Then, whenever it makes sense, one has

$$
\begin{aligned}
& \alpha(c f)=|c| \alpha(f), \quad \forall c \in \mathbb{R} ; \\
& \alpha(f)-\alpha(g) \leq \alpha(f+g) \leq \alpha(f)+\alpha(g) ; \\
& \omega(c f)=|c| \omega(f), \quad \forall c \in \mathbb{R} ; \\
& \omega(f)-\alpha(g) \leq \omega(f+g) \leq \omega(f)+\alpha(g) .
\end{aligned}
$$

Proposition 4.4. Let $f: X \rightarrow Y$ and $g: Y \rightarrow Z$ be two $\alpha$-admissible maps between metric spaces. Then, whenever it makes sense, one has

$$
\begin{aligned}
& \alpha(g \circ f) \leq \alpha(g) \alpha(f) ; \\
& \omega(g \circ f) \geq \omega(g) \omega(f) .
\end{aligned}
$$

In the case of a linear operator, we get the following properties.

Proposition 4.5. Let $L: E \rightarrow F$ be a bounded linear operator between Banach spaces and denote by $L^{*}$ its adjoint. Then

$$
\begin{aligned}
& \alpha(L) \leq\|L\| ; \\
& \omega(L)>0 \text { if and only if } L \text { is left semi-Fredholm; } \\
& \omega\left(L^{*}\right)>0 \text { if and only if } L \text { is right semi-Fredholm. }
\end{aligned}
$$

As a consequence of Proposition 4.5 one gets that a bounded linear operator $L$ is Fredholm if and only if $\omega(L)>0$ and $\omega\left(L^{*}\right)>0$.

In the sequel, each time we consider the product $E_{1} \times E_{2}$ of two Banach spaces $\left(E_{1},\|\cdot\|_{1}\right)$ and $\left(E_{2},\|\cdot\|_{2}\right)$, we shall tacitly assume that the norm in this product is defined by

$$
\left\|\left(x_{1}, x_{2}\right)\right\|=\max \left\{\left(\left\|x_{1}\right\|_{1},\left\|x_{2}\right\|_{2}\right)\right\} .
$$

Remark 4.6. We point out that, given the product $E_{1} \times E_{2}$ of two Banach spaces, the two natural projections $\pi_{1}$ and $\pi_{2}$ onto the factors spaces are both nonexpansive and, consequently, they are also $\alpha$-nonexpansive (i.e. $\alpha$ Lipschitz with constant 1). Actually, if one of the two spaces, say $E_{2}$, is finite dimensional, then the first projection is $\alpha$-conservative; that is, for any bounded subset $A \subseteq E_{1} \times E_{2}$, one has $\alpha\left(\pi_{1}(A)\right)=\alpha(A)$. This can be easily deduced from the fact that $\pi_{1}$ is $\alpha$-nonexpansive and from the following property (see e.g. [20]): If $A_{1} \subseteq E_{1}$ and $A_{2} \subseteq E_{2}$ are bounded sets, then $\alpha\left(A_{1} \times A_{2}\right)=\max \left\{\alpha\left(A_{1}\right), \alpha\left(A_{2}\right)\right\}$. 
Let $f: X \rightarrow Y$ be a continuous map between metric spaces, and fix $p \in X$. We recall the definitions of $\alpha_{p}(f)$ and $\omega_{p}(f)$ given in [10]. Roughly speaking, these numbers are the local analogues of $\alpha(f)$ and $\omega(f)$. Let $B(p, r) \subseteq X$ denote the open ball in $X$ centered at $p$ with radius $r>0$. Define

$$
\alpha_{p}(f)=\lim _{r \rightarrow 0} \alpha\left(\left.f\right|_{B(p, r)}\right), \quad \omega_{p}(f)=\lim _{r \rightarrow 0} \omega\left(\left.f\right|_{B(p, r)}\right) .
$$

Notice that $f$, being continuous, is locally $\alpha$-admissible. More precisely, any point of $X$ admits a neighborhood whose image under $f$ is bounded. Thus, the two (extended real valued) functions of $r, \alpha\left(\left.f\right|_{B(p, r)}\right)$ and $\omega\left(\left.f\right|_{B(p, r)}\right)$, are defined in a right neighborhood of 0 and, as one can easily check, they are monotone. Therefore the definitions of the above two numbers are well posed.

Clearly, if $f$ is $\alpha$-admissible, then $\alpha_{p}(f) \leq \alpha(f)$ and $\omega_{p}(f) \geq \omega(f)$.

With only minor changes, it is easy to show that the main properties of $\alpha$ and $\omega$ hold for $\alpha_{p}$ and $\omega_{p}$ (see [10] for details).

In the case of a bounded linear operator $L: E \rightarrow F$, the numbers $\alpha_{p}(L)$ and $\omega_{p}(L)$ do not depend on the point $p$ and coincide with $\alpha(L)$ and $\omega(L)$, respectively.

Proposition $4.7([10])$. Let $f: \Omega \rightarrow F$ be of class $C^{1}$ on an open subset of $E$. Then, for any $p \in \Omega$ we have $\alpha_{p}(f)=\alpha\left(f^{\prime}(p)\right)$ and $\omega_{p}(f)=\omega\left(f^{\prime}(p)\right)$.

If $f: \Omega \rightarrow F$ is a Fredholm map, as a straightforward consequence of Propositions 4.5 and 4.7 , we obtain $\omega_{p}(f)>0$ for any $p \in \Omega$.

Based on the two numbers $\alpha_{p}$ and $\omega_{p}$, we can now recall the definition of $\alpha$-Fredholm map.

Definition 4.8. An $\alpha$-Fredholm map $f: \Omega \rightarrow F$ is of the form $f=g-k$, where $g$ is a Fredholm map of index zero, $k$ is continuous and $\alpha_{p}(k)<\omega_{p}(g)$ for every $p \in \Omega$.

In particular, a quasi-Fredholm map $f=g-k$ is also $\alpha$-Fredholm since $\omega_{p}(g)>0$ and $\alpha_{p}(k)=0$ for any $p$ in the domain of $f$.

Let us now recall the definitions of admissible $\alpha$-Fredholm triple and of (oriented) $\alpha$-Fredholm homotopy.

Definition 4.9. Let $g: \Omega \rightarrow F$ be an oriented Fredholm map of index zero, $k: \Omega \rightarrow F$ a continuous map, and $U$ an open subset of $\Omega$ such that $\alpha_{p}(k)<\omega_{p}(g)$ for any $p \in U$. The triple $(g, U, k)$ is said to be an admissible $\alpha$-Fredholm triple if the solution set $\{x \in U: g(x)=k(x)\}$ is compact.

Definition 4.10. Let $U$ be an open subset of $E$ and $H: U \times[0,1] \rightarrow F$ a continuous map of the form

$$
H(x, s)=G(x, s)-K(x, s) .
$$

We say that $H$ is an $\alpha$-Fredholm homotopy if the following conditions hold:

- $G$ is $C^{1}$; 
- for any $s \in[0,1]$ the partial map $G_{s}(\cdot)=G(\cdot, s)$ is Fredholm of index zero;

- for any pair $(x, s) \in U \times[0,1]$ we have $\alpha_{(x, s)}(K)<\omega_{(x, s)}(G)$.

An orientation of $H=G-K$ is an orientation of the partial derivative

$$
\partial_{1} G: U \times[0,1] \rightarrow \Phi_{0}(E, F), \quad(x, s) \mapsto\left(G_{s}\right)^{\prime}(x),
$$

according to Definition 3.4.

The $\alpha$-Fredholm degree is an integer valued map,

$$
(g, U, k) \mapsto \operatorname{deg}_{\alpha \mathrm{F}}(g, U, k),
$$

defined on the set of the admissible $\alpha$-Fredholm triples. Its construction, given in [2] (see [3] for a further extension), is based on the notion of degree for quasi-Fredholm maps, and its properties are analogous to the ones of the Leray-Schauder degree. Here we mention the principal ones:

i) (Normalization) Let the identity $I$ of $E$ be naturally oriented. Then

$$
\operatorname{deg}_{\alpha \mathrm{F}}(I, E, 0)=1 .
$$

ii) (Additivity) If $(g, U, k)$ is an admissible $\alpha$-Fredholm triple and $U_{1}$, $U_{2}$ are two disjoint open subsets of $U$ such that the set $\{x \in U$ : $g(x)=k(x)\}$ is contained in $U_{1} \cup U_{2}$, then

$$
\operatorname{deg}_{\alpha \mathrm{F}}(g, U, k)=\operatorname{deg}_{\alpha \mathrm{F}}\left(g, U_{1}, k\right)+\operatorname{deg}_{\alpha \mathrm{F}}\left(g, U_{2}, k\right) .
$$

iii) (Existence) Let $(g, U, k)$ be admissible. If

$$
\operatorname{deg}_{\alpha \mathrm{F}}(g, U, k) \neq 0,
$$

then the equation $g(x)=k(x)$ has a solution in $U$.

iv) (Excision) If $(g, U, k)$ is an admissible triple and $V$ is an open subset of $U$ containing $\{x \in U: g(x)=k(x)\}$, then

$$
\operatorname{deg}_{\alpha \mathrm{F}}(g, V, k)=\operatorname{deg}_{\alpha \mathrm{F}}(g, U, k) .
$$

v) (Homotopy invariance) Let $H: U \times[0,1] \rightarrow F$ be an oriented $\alpha$ Fredholm homotopy of the form $H(x, s)=G(x, s)-K(x, s)$. If the set $H^{-1}(0)$ is compact, then $\operatorname{deg}_{\alpha \mathrm{F}}\left(G_{s}, U, K_{s}\right)$ does not depend on $s \in[0,1]$.

In addition we have the following compatibility properties (see $[2,4]$ ):

vi) ( $q F$-compatibility) If $(g, U, k)$ is an admissible $\alpha$-Fredholm triple with $k$ locally compact, then

$$
\operatorname{deg}_{\alpha \mathrm{F}}(g, U, k)=\operatorname{deg}_{\mathrm{qF}}(g-k, U, 0),
$$

where the orientation of $g-k$ is the one transported along the homotopy $(x, s) \mapsto g(x)-s k(x)$.

vii) (LS-compatibility) Let $(I, U, k)$ be a triple in which $I$ is the identity of $E, U$ is a bounded open subset of $E$, and $k: \bar{U} \rightarrow E$ is a completely continuous map. If $I$ is naturally oriented and $0 \notin(I-k)(\partial U)$, then

$$
\operatorname{deg}_{\alpha \mathrm{F}}(I, U, k)=\operatorname{deg}_{\mathrm{LS}}(I-k, U, 0),
$$


where $\operatorname{deg}_{\mathrm{LS}}(I-k, U, 0)$ denotes the Leray-Schauder degree of $I-k$ in $U$ with respect to $0 \in E$.

The following lemma will help us to compute the number $\omega$ of a homotopy in which a Fredholm linear operator is involved.

Lemma 4.11. Given a bounded linear operator $L: E \rightarrow F$, define $H: E \times$ $\mathbb{R}^{k} \rightarrow F$ by $(x, s) \mapsto L x$. Then, $\omega(H)=\omega(L)$. Consequently, $H$ being linear, one has $\omega_{(x, s)}(H)=\omega(L)$ for any $(x, s) \in E \times \mathbb{R}^{k}$.

Proof. Define $J: E \rightarrow E \times \mathbb{R}^{k}$ by $x \mapsto(x, 0)$, and observe that $H \circ J=L$. Since $J$ is an isometry, we have $\alpha(J)=1$. Therefore, applying property (4.12), we get

$$
\omega(H) \leq \omega(L) .
$$

It remains to prove the reverse inequality. Notice that $H=L \circ \pi_{1}$, where $\pi_{1}: E \times \mathbb{R}^{k} \rightarrow E$ is the projection onto the first factor, which, according to Remark 4.6, is $\alpha$-conservative. Thus, again by property (4.12), we obtain

$$
\omega(H) \geq \omega(L),
$$

and the equality $\omega(H)=\omega(L)$ follows.

Let $f_{0}, f_{1}: U \rightarrow F$ be two $\alpha$-Lipschitz maps defined on an open subset of $E$. The following lemma will help us to evaluate the numbers $\alpha$ and $\alpha_{p}$ of a homotopy of the type $(x, s) \mapsto(1-s) f_{0}(x)+s f_{1}(x)$, hereafter called straight line homotopy (joining $f_{0}$ and $f_{1}$ ).

Lemma 4.12. Let $U$ be an open subset of $E$ and $\mathcal{S}$ a compact subset of $\mathbb{R}^{k}$. Given an $\alpha$-Lipschitz map $f: U \rightarrow F$ and a continuous function $\sigma: \mathcal{S} \rightarrow \mathbb{R}$, define

$$
H: U \times \mathcal{S} \rightarrow F \quad \text { by } H(x, s)=\sigma(s) f(x) .
$$

Then $\alpha(H)=\max \{|\sigma(s)|: s \in \mathcal{S}\} \alpha(f)$. Consequently, given any $(x, s) \in$ $U \times \mathcal{S}$, one has $\alpha_{(x, s)}(H)=|\sigma(s)| \alpha_{x}(f)$.

Proof. Let $\bar{s} \in \mathcal{S}$ be such that $|\sigma(\bar{s})|=\max \{|\sigma(s)|: s \in \mathcal{S}\}$. We need to prove that $\alpha(H)=|\sigma(\bar{s})| \alpha(f)$. Define the isometry $\bar{J}: U \rightarrow U \times \mathcal{S}$ by $\bar{J}(x)=(x, \bar{s})$. We have $H \circ \bar{J}=\sigma(\bar{s}) f$. Therefore, applying properties (4.7) and (4.11), and taking into account that $\alpha(\bar{J})=1$, we get

$$
\alpha(H) \geq|\sigma(\bar{s})| \alpha(f) .
$$

It remains to prove the reverse inequality. Given a bounded subset $A$ of $U \times \mathcal{S}$, we actually have $A \subseteq \pi_{1}(A) \times \mathcal{S}$, where $\pi_{1}$ is the projection of $U \times \mathcal{S}$ onto $U$. Thus, $H(A) \subseteq \sigma(\mathcal{S}) f\left(\pi_{1}(A)\right)$. Therefore, applying property (4.5), we obtain

$$
\alpha(H(A)) \leq \alpha\left(\sigma(\mathcal{S}) f\left(\pi_{1}(A)\right)\right)=|\sigma(\bar{s})| \alpha\left(f\left(\pi_{1}(A)\right)\right) \leq|\sigma(\bar{s})| \alpha(f) \alpha\left(\pi_{1}(A)\right) .
$$

Recalling that the projection $\pi_{1}$ is $\alpha$-nonexpansive (see Remark 4.6), we get

$$
\alpha(H(A)) \leq|\sigma(\bar{s})| \alpha(f) \alpha(A),
$$


which implies

$$
\alpha(H) \leq|\sigma(\bar{s})| \alpha(f) .
$$

Thus $\alpha(H)=\max |\sigma| \alpha(f)$ and, consequently, $\alpha_{(x, s)}(H)=|\sigma(s)| \alpha_{x}(f)$.

We conclude this section by adding a new property (ignored in $[2,3]$ ) that we will utilize in the proof of Proposition 5.4.

Lemma 4.13. The $\alpha$-Fredholm degree has the following property:

viii) (Sign) Given $L, T \in L(E, F)$, assume that $L-T$ is invertible and that $\omega(L)>\alpha(T)$. Then $L-s T \in \Phi_{0}(E, F)$ for all $s \in[0,1]$ and

$$
\operatorname{deg}_{\alpha \mathrm{F}}(L, E, T)=\operatorname{deg}_{\alpha \mathrm{F}}(L-T, E, 0)=\operatorname{sign}(L-T),
$$

provided that $L$ and $L-T$ are oriented with orientations linked through the straight line homotopy.

Proof. According to properties (4.7) and (4.10), for any $s \in[0,1]$ one has

$$
\omega(L-s T) \geq \omega(L)-\alpha(s T)=\omega(L)-s \alpha(T) \geq \omega(L)-\alpha(T)>0 .
$$

Thus, because of Proposition 4.5, $L-s T$ is (left) semi-Fredholm for all $s \in[0,1]$. Since $L-T$ is invertible, one has $L-T \in \Phi_{0}(E, F)$. Consequently, recalling that the function ind: $\bar{\Phi}(E, F) \rightarrow \overline{\mathbb{Z}}$ is locally constant, we get

$$
L-s T \in \Phi_{0}(E, F), \quad \forall s \in[0,1] .
$$

Now define $G_{L}, G_{T}, K: E \times[0,1] \rightarrow F$ by

$$
G_{L}(x, s)=L x, \quad G_{T}(x, s)=s T x, \quad K(x, s)=(1-s) T x .
$$

Put $G=G_{L}-G_{T}, H=G-K$ and choose any $(x, s) \in E \times[0,1]$. Applying, respectively, Lemma 4.11 and Lemma 4.12 to $G_{L}$ and $G_{T}$, we obtain $\omega_{(x, s)}\left(G_{L}\right)=\omega(L)$ and $\alpha_{(x, s)}\left(G_{T}\right)=s \alpha(T)$. Hence, property (4.10) implies

$$
\omega_{(x, s)}(G)=\omega_{(x, s)}\left(G_{L}-G_{T}\right) \geq \omega_{(x, s)}\left(G_{L}\right)-\alpha_{(x, s)}\left(G_{T}\right)=\omega(L)-s \alpha(T) .
$$

Analogously, one has $\alpha_{(x, s)}(K)=(1-s) \alpha(T)$. Consequently,

$$
\omega_{(x, s)}(G)-\alpha_{(x, s)}(K) \geq(\omega(L)-s \alpha(T))-(1-s) \alpha(T)=\omega(L)-\alpha(T)>0 .
$$

Thus $H=G-K$ is an $\alpha$-Fredholm homotopy.

Now, according to Theorem 3.7, we orient $G$ by choosing one of the two possible orientations of the partial map $G_{0}=L$. Hence, $H$ is an oriented $\alpha$ Fredholm homotopy (as in Definition 4.10). Moreover, $G_{0}=L$ and $G_{1}=L-$ $T$ are oriented with orientations linked through the straight line homotopy.

Observe that $H_{s}=L-T$ for all $s \in[0,1]$. Therefore, $H^{-1}(0)$ is the compact set $\{0\} \times[0,1]$. Consequently, by the homotopy invariance property, we get

$$
\operatorname{deg}_{\alpha \mathrm{F}}(L, E, T)=\operatorname{deg}_{\alpha \mathrm{F}}(L-T, E, 0)
$$

and by the $\mathrm{qF}$-compatibility property, one has

$$
\operatorname{deg}_{\alpha \mathrm{F}}(L-T, E, 0)=\operatorname{deg}_{\mathrm{qF}}(L-T, E, 0) .
$$


Finally, the sign property of the $\mathrm{qF}$-degree implies

$$
\operatorname{deg}_{\mathrm{qF}}(L-T, E, 0)=\operatorname{sign}(L-T),
$$

and we get the assertion.

\section{Results}

Let $E$ and $F$ be two real Banach spaces, and let $\Omega \subseteq E$ be an open subset such that $0 \in \Omega$. Consider the system

$$
\left\{\begin{array}{l}
L x+\varepsilon N(x)=\lambda C x \\
x \in \partial \Omega
\end{array}\right.
$$

where $L: E \rightarrow F$ and $C: E \rightarrow F$ are bounded linear operators, $N: \bar{\Omega} \rightarrow F$ is continuous and locally $\alpha$-Lipschitz, and $\lambda$ and $\varepsilon$ are real parameters.

A solution of (5.1) is a triple $(x, \varepsilon, \lambda) \in \partial \Omega \times \mathbb{R} \times \mathbb{R}$ satisfying the equation $L x+\varepsilon N(x)=\lambda C x$. The first and third elements of a solution $(x, \varepsilon, \lambda)$ will be called, respectively, a $\partial \Omega$-eigenvector and a $\partial \Omega$-eigenvalue (of the equation $L x+\varepsilon N(x)=\lambda C x)$. A particular case is when $\Omega=\{x \in E: \gamma(x)<1\}$, with $\gamma: E \rightarrow \mathbb{R}$ any continuous norm on $E$ not necessarily equivalent to the Banach norm $\|\cdot\|$. In this case a $\partial \Omega$-eigenvector is also called a unit eigenvector (as in $[14,15]$ ).

We assume that $L$ is Fredholm of index zero with nontrivial kernel and that

$$
\operatorname{Im} L \oplus C(\operatorname{Ker} L)=F .
$$

As a consequence, there exists $\sigma>0$ such that $L-\lambda C$ is invertible if $0<|\lambda| \leq \sigma$. In fact, according to the decompositions $E=E_{1} \oplus \operatorname{Ker} L$ and $F=\operatorname{Im} L \oplus C(\operatorname{Ker} L)$, write $L-\lambda C$ in a block-matrix form as

$$
L-\lambda C=\left(\begin{array}{cc}
L_{11}-\lambda C_{11} & 0 \\
-\lambda C_{21} & -\lambda C_{22}
\end{array}\right)
$$

where $L_{11}: E_{1} \rightarrow \operatorname{Im} L$ and $C_{22}: \operatorname{Ker} L \rightarrow C(\operatorname{Ker} L)$ are isomorphisms. Thus, so is $L_{11}-\lambda C_{11}$ if $\lambda$ is sufficiently small. Consequently, for $\lambda$ small and different from zero, given any $\left(y_{1}, y_{2}\right) \in \operatorname{Im} L \times C(\operatorname{Ker} L)$, the system

$$
\left\{\begin{aligned}
L_{11} x_{1}-\lambda C_{11} x_{1} & =y_{1} \\
-\lambda C_{21} x_{1}-\lambda C_{22} x_{2} & =y_{2}
\end{aligned}\right.
$$

has one and only one solution $\left(x_{1}, x_{2}\right) \in E_{1} \times \operatorname{Ker} L$. This means that $\lambda=0$ is an isolated eigenvalue for the problem $L x=\lambda C x$.

The following remark will be useful in order to check that condition (5.2) holds true for some examples in Section 6.

Remark 5.1. Condition (5.2) implies $\operatorname{dim} C(\operatorname{Ker} L)=\operatorname{codim} \operatorname{Im} L$. Thus, taking into account that codim $\operatorname{Im} L=\operatorname{dim} \operatorname{Ker} L,(5.2)$ is equivalent to

$$
\operatorname{Im} L+C(\operatorname{Ker} L)=F \text {. }
$$


Moreover, it is also equivalent to

$$
(u \in \operatorname{Ker} L) \wedge(C u \in \operatorname{Im} L) \Longrightarrow u=0 .
$$

In fact, if (5.4) holds, the restriction of $C$ to $\operatorname{Ker} L$ is clearly injective and, consequently, $C(\operatorname{Ker} L)$ has the same dimension as $\operatorname{Ker} L$, which equals the codimension of $\operatorname{Im} L$. Thus, (5.2) is verified, since (5.4) implies also $\operatorname{Im} L \cap$ $C(\operatorname{Ker} L)=\{0\}$. The converse implication is true because, if (5.2) holds, one has $\operatorname{dim} C(\operatorname{Ker} L)$ equals codim $\operatorname{Im} L$, which is the same as $\operatorname{dim} \operatorname{Ker} L$. Hence $C$ is one-to-one on $\operatorname{Ker} L$, and this implies (5.4) since $\operatorname{Im} L \cap C(\operatorname{Ker} L)=\{0\}$.

Notice that a triple of the type $(x, 0,0) \in E \times \mathbb{R} \times \mathbb{R}$ is a solution of (5.1) if and only if $x$ belongs to the set $\Sigma:=\partial \Omega \cap \operatorname{Ker} L$. These distinguished solutions (the ones with $(\varepsilon, \lambda)=(0,0)$ ) will be called trivial and, consequently, all the others will be said nontrivial. Analogously, $\Sigma$ will be called the set of the trivial $\partial \Omega$-eigenvectors (of (5.1)). Due to this distinction, it makes sense to introduce the following definition.

Definition 5.2 (Bifurcation points). A trivial $\partial \Omega$-eigenvector $x_{0} \in \Sigma$ will be called a bifurcation point of system (5.1) if any neighborhood of $\left(x_{0}, 0,0\right)$ contains nontrivial solutions of (5.1).

Let $x_{0} \in \Sigma$ be a bifurcation point of (5.1). Since, as already observed, $L-\lambda C$ is invertible for $|\lambda|>0$ small, one gets that any nontrivial solution $(x, \varepsilon, \lambda)$ of $(5.1)$ sufficiently close to the bifurcation triple $\left(x_{0}, 0,0\right)$ must have $\varepsilon \neq 0$.

The proof of the following result can be found in [15] (see also [9, Corollary 5.1] for a more general version).

Lemma 5.3 (On the sign-jump). Let $b>0$ be such that $L-\lambda C$ is invertible for $0<|\lambda| \leq b$. Then, given any one of the two orientations (in the sense of Definition 3.4) of the map

$$
h:[-b, b] \rightarrow \Phi_{0}(E, F), \quad h(\lambda)=L-\lambda C,
$$

sign $h(\lambda)$ is constant in each one of the intervals $[-b, 0)$ and $(0, b]$, and changes crossing $\lambda=0$ if and only if $\operatorname{Ker} L$ is odd dimensional.

In Proposition 5.4 below, which is crucial in obtaining our main results on the persistence of the eigenvalues and eigenvectors, the nonlinear map $N$ is assumed to be defined and $\alpha$-Lipschitz on the closure $\bar{U}$ of a bounded open subset $U$ of $E$.

Proposition 5.4. Let $L, C: E \rightarrow F$ be as above, let $U$ be a bounded open subset of $E$ containing the origin, and let $N: \bar{U} \rightarrow F$ be continuous and $\alpha$-Lipschitz. Assume that $\operatorname{Ker} L$ is odd dimensional. Then, there exist $a>0$ and $b>0$ such that

(1) the set $\mathcal{K}=\{(x, \varepsilon, \lambda) \in \partial U \times[-a, a] \times[-b, b]: L x+\varepsilon N(x)=\lambda C x\}$ is compact; 
(2) for any $\varepsilon \in[-a, a]$ there exist $\lambda \in[-b, b]$ and $x \in \partial U$ such that $(x, \varepsilon, \lambda) \in \mathcal{K}$

(3) $(x, 0, \lambda) \in \mathcal{K}$ if and only if $\lambda=0$ and $x \in \partial U \cap \operatorname{Ker} L$.

Proof. Notice that, by Proposition 4.5, $\alpha(C)$ is finite since $C$ is bounded, and $\omega(L)>0$ since $L$ is Fredholm. Let $a>0$ and $b>0$ be such that $a \alpha(N)+b \alpha(C)<\omega(L)$. Consider the rectangle $\mathcal{R}:=[-a, a] \times[-b, b]$ and define $H=G-K: \bar{U} \times \mathcal{R} \rightarrow F$ by

$$
G(x, \varepsilon, \lambda)=L x, \quad K(x, \varepsilon, \lambda)=\lambda C x-\varepsilon N(x) .
$$

We claim that the map $H$ is proper. To see this observe first that, applying Lemma 4.11, we get $\omega(G)=\omega(L)$. Now, define $K_{N}, K_{C}: \bar{U} \times \mathcal{R} \rightarrow F$ by

$$
K_{N}(x, \varepsilon, \lambda)=-\varepsilon N(x), \quad K_{C}(x, \varepsilon, \lambda)=\lambda C x,
$$

so that $K=K_{N}+K_{C}$. By Lemma 4.12, taking $\sigma(\varepsilon, \lambda)=\varepsilon$ for $K_{N}$ and $\sigma(\varepsilon, \lambda)=\lambda$ for $K_{C}$, we obtain $\alpha\left(K_{N}\right)=a \alpha(N)$ and $\alpha\left(K_{C}\right)=b \alpha(C)$. Hence, recalling property (4.8), we have

$$
\alpha(K) \leq \alpha\left(K_{N}\right)+\alpha\left(K_{C}\right)=a \alpha(N)+b \alpha(C) .
$$

Consequently, using property (4.10), we get

$$
\omega(H) \geq \omega(G)-\alpha(K) \geq \omega(L)-a \alpha(N)-b \alpha(C)>0 .
$$

Therefore, the map $H$ is proper, being $\alpha$-coercive on the bounded complete set $\bar{U} \times \mathcal{R}$ (see Remark 4.2). This implies, in particular, that the set

$$
H^{-1}(0)=\{(x, \varepsilon, \lambda) \in \bar{U} \times \mathcal{R}: L x+\varepsilon N(x)-\lambda C x=0\}
$$

is compact. Consequently, its closed subset

$$
\mathcal{K}=\{(x, \varepsilon, \lambda) \in \partial U \times \mathcal{R}: L x+\varepsilon N(x)-\lambda C x=0\}
$$

is compact as well. This proves assertion (1).

Further, by projecting $\mathcal{K}$ into $\mathcal{R}$, it follows that also

$$
\Gamma=\{(\varepsilon, \lambda) \in \mathcal{R}: L x+\varepsilon N(x)-\lambda C x=0 \text { for some } x \in \partial U\}
$$

is a compact set.

Now, choose one of the two orientations of $L$. We claim that, because of the inequality $a \alpha(N)+b \alpha(C)<\omega(L)$, for any $(\varepsilon, \lambda)$ in the relatively open complement $\mathcal{R} \backslash \Gamma$ of $\mathcal{R}$, the triple $(L, U, \lambda C-\varepsilon N)$ is $\alpha$-Fredholm admissible. In fact, observe first that, for $(\varepsilon, \lambda) \in \mathcal{R} \backslash \Gamma$, the compact set

$$
H_{(\varepsilon, \lambda)}^{-1}(0)=\{x \in \bar{U}: H(x, \varepsilon, \lambda)=0\}
$$

is contained in $U$. Moreover, the properties of $\omega_{p}, \alpha_{p}$ and $\alpha$ imply that for any $x \in U$ one has

$$
\alpha_{x}(\lambda C-\varepsilon N) \leq \alpha(\lambda C-\varepsilon N) \leq a \alpha(N)+b \alpha(C)<\omega(L)=\omega_{x}(L),
$$

proving the claim.

Now, given any $(x, \varepsilon, \lambda) \in U \times \mathcal{R}$, we have

$$
\omega_{(x, \varepsilon, \lambda)}(G)-\alpha_{(x, \varepsilon, \lambda)}(K) \geq \omega(G)-\alpha(K)>0,
$$


the second term being positive because of equation (5.5). Thus, from the homotopy invariance property of the $\alpha$-Fredholm degree we deduce that the integer valued function

$$
(\varepsilon, \lambda) \in \mathcal{R} \backslash \Gamma \mapsto \operatorname{deg}_{\alpha \mathrm{F}}(L, U, \lambda C-\varepsilon N)
$$

is locally constant.

Now, as already pointed out, the condition (5.2) on $L$ and $C$ implies that $L-\lambda C$ is invertible for small $|\lambda| \neq 0$. Hence, without loss of generality, we may assume that this happens for $0<|\lambda| \leq b$. Thus, since $0 \notin \partial U$, the two points $(0, \pm b)$ belong to $\mathcal{R} \backslash \Gamma$. We claim that

$$
\operatorname{deg}_{\alpha \mathrm{F}}(L, U, b C) \neq \operatorname{deg}_{\alpha \mathrm{F}}(L, U,-b C) .
$$

In fact, from Lemma 4.13 and the excision property of the $\alpha$-Fredholm degree, we obtain

$$
\operatorname{deg}_{\alpha \mathrm{F}}(L, U, b C)=\operatorname{sign}(L-b C) \text { and } \operatorname{deg}_{\alpha \mathrm{F}}(L, U,-b C)=\operatorname{sign}(L+b C),
$$

provided that the orientations of $L-b C$ and $L+b C$ are linked to the orientation of $L$ through the straight line homotopy. Now observe that the orientations of these two maps are also linked one to the other through the straight line homotopy. Therefore, Lemma 5.3 applies, getting

$$
\operatorname{sign}(L-b C) \neq \operatorname{sign}(L+b C),
$$

which, recalling (5.7), implies our claim (5.6).

Hence, the two points $(0, b)$ and $(0,-b)$ lie in different connected components of $\mathcal{R} \backslash \Gamma$ and, by taking $a$ smaller if necessary, we may assume that also the up and down edges of the rectangle $\mathcal{R}$ lie in different components of $\mathcal{R} \backslash \Gamma$. This proves that for any $\varepsilon \in[-a, a]$ there exists $\lambda \in[-b, b]$ such that $(\varepsilon, \lambda) \in \Gamma$, which is the assertion $(2)$.

Assertion (3) follows from the fact that $L-\lambda C$ is invertible for $0<|\lambda| \leq$ $b$.

In what follows, given $x \in E$ and $A \subseteq E$, $\operatorname{dist}(x, A)$ denotes the distance from the point $x$ to the set $A$. Namely, $\operatorname{dist}(x, A)=\inf _{a \in A}\|x-a\|$.

Theorem 5.5 (Persistence of the eigenvalues). Let $L, C, N$ and $\Omega$ be as in (5.1). Assume that the set $\Sigma=\partial \Omega \cap \operatorname{Ker} L$ of the trivial $\partial \Omega$-eigenvectors is nonempty and compact, and that $\operatorname{Ker} L$ is odd dimensional. Then, given $c>0$ sufficiently small, there exist $a>0$ and $b>0$ such that:

(1) for any $\varepsilon \in[-a, a]$, the set of eigenvalues

$$
\begin{aligned}
\Gamma_{\varepsilon}= & \{\lambda \in[-b, b]: L x+\varepsilon N(x)=\lambda C x \text { for some } x \in \partial \Omega \text { with } \\
& \operatorname{dist}(x, \Sigma)<c\}
\end{aligned}
$$

is nonempty;

(2) the multivalued eigenvalue map $\varepsilon \in[-a, a] \vdash \Gamma_{\varepsilon}$ is upper semicontinuous;

(3) $\Gamma_{0}=\{0\}$. 
Proof. Since $N$ is locally $\alpha$-Lipschitz, there exists a bounded open neighborhood $W$ of the compact set $\Sigma \cup\{0\}$ such that $N$ is (globally) $\alpha$-Lipschitz on $\overline{\Omega \cap W}$. Consequently, we can apply Proposition 5.4 with $U=\Omega \cap W$. Therefore, there exists $\mathcal{R}=[-a, a] \times[-b, b]$ such that the three assertions of the above proposition hold true. Observe that these assertions will remain true even taking a smaller rectangle (as we shall do later).

Now, choose any $c>0$ such that the open set $V_{c}=\{x \in E: \operatorname{dist}(x, \Sigma)<$ $c\}$ is contained in $W$. The existence of such a set is ensured by the fact that $\Sigma$ is nonempty, compact, and contained in $W$.

By assertion (2) of Proposition 5.4 we know that, given any $\varepsilon \in[-a, a]$, there exist $\lambda \in[-b, b]$ and $x \in \partial U$ such that $(x, \varepsilon, \lambda) \in \mathcal{K}$. Therefore, in order to prove the assertion (1) of the theorem, it is enough to show that, if $(x, \varepsilon, \lambda) \in \mathcal{K}$, then $x$ belongs actually to $\partial \Omega \cap V_{c}$ (by reducing $a$ and $b$ if needed). To see this observe that, since $\mathcal{K}$ is compact, Remark 2.1 ensures that the multivalued map $(\varepsilon, \lambda) \in \mathcal{R} \longmapsto \mathcal{K}_{(\varepsilon, \lambda)}$ is upper semicontinuous (here $\mathcal{K}_{(\varepsilon, \lambda)} \subseteq \partial U$ denotes the slice of $\mathcal{K}$ at $\left.(\varepsilon, \lambda)\right)$. On the other hand, the set $\mathcal{K}_{(0,0)}$ coincides with the subset $\Sigma$ of $V_{c}$. Hence, by reducing $a$ and $b$ if necessary, we get $\mathcal{K}_{(\varepsilon, \lambda)} \subseteq V_{c}$ for all $(\varepsilon, \lambda) \in \mathcal{R}$. That is, we may assume that $\mathcal{R}$ is such that

$$
\mathcal{K}=\left\{(x, \varepsilon, \lambda) \in\left(\partial U \cap V_{c}\right) \times \mathcal{R}: L x+\varepsilon N(x)=\lambda C x\right\} .
$$

Now observe that

$$
\partial(\Omega \cap W) \cap W=\partial \Omega \cap W .
$$

Thus, since $\Omega \cap W=U$ and $V_{c} \subseteq W$, by intersecting both members of the last equality with $V_{c}$, we get $\partial U \cap V_{c}=\partial \Omega \cap V_{c}$. Consequently,

$$
\mathcal{K}=\left\{(x, \varepsilon, \lambda) \in\left(\partial \Omega \cap V_{c}\right) \times \mathcal{R}: L x+\varepsilon N(x)=\lambda C x\right\},
$$

and this, as previously observed, implies assertion (1).

Assertion (2), according to Remark 2.1, is a consequence of the compactness of the set

$$
\Gamma=\left\{(\varepsilon, \lambda) \in \mathcal{R}: L x+\varepsilon N(x)=\lambda C x \text { for some } x \in \partial \Omega \cap V_{c}\right\},
$$

which, because of (5.8), is the projection of $\mathcal{K}$ into the rectangle $\mathcal{R}$, as well as the graph of the multivalued map $\varepsilon \in[-a, a] \mapsto \Gamma_{\varepsilon}$.

Assertion (3) holds since in Proposition 5.4 we have chosen $b$ such that $L-\lambda C$ is invertible for $0<|\lambda| \leq b$.

As in the proof of Theorem 5.5, hereafter, given $c>0$, we put

$$
V_{c}=\{x \in E: \operatorname{dist}(x, \Sigma)\},
$$

where $\Sigma=\partial \Omega \cap \operatorname{Ker} L$.

Theorem 5.6 (Persistence of the eigenvectors). Let $L, C, N$ and $\Omega$ be as in (5.1). Assume that $\Sigma=\partial \Omega \cap \operatorname{Ker} L$ is nonempty, compact, and that $\operatorname{Ker} L$ is odd dimensional. Then, given $c>0$ sufficiently small, there exist $a>0$ and $b>0$ such that: 
(1) for any $\varepsilon \in[-a, a]$, the set of eigenvectors

$\Xi_{\varepsilon}=\left\{x \in \partial \Omega \cap V_{c}: L x+\varepsilon N(x)=\lambda C x\right.$ for some $\left.\lambda \in[-b, b]\right\}$

is nonempty;

(2) the multivalued eigenvector map $\varepsilon \in[-a, a] \vdash \Xi_{\varepsilon}$ is upper semicontinuous;

(3) $\Xi_{0}=\Sigma$.

Proof. Let $a, b, c, \mathcal{R}=[-a, a] \times[-b, b]$ and the compact set

$$
\mathcal{K}=\left\{(x, \varepsilon, \lambda) \in\left(\partial \Omega \cap V_{c}\right) \times \mathcal{R}: L x+\varepsilon N(x)=\lambda C x\right\}
$$

be as in the proof of Theorem 5.5.

Assertion (1) is a direct consequence of the homologous one in Theorem 5.5 .

Assertion (2), because of Remark 2.1, follows from the compactness of the set

$\Xi=\left\{(x, \varepsilon) \in\left(\partial \Omega \cap V_{c}\right) \times[-a, a]: L x+\varepsilon N(x)=\lambda C x\right.$ for some $\left.\lambda \in[-b, b]\right\}$, which is the projection of $\mathcal{K}$ into $\left(\partial \Omega \cap V_{c}\right) \times[-a, a]$, as well as the graph of the multivalued map $\varepsilon \in[-a, a] \longmapsto \Xi_{\varepsilon}$. $b$.

Assertion (3) holds since $b$ is such that $L-\lambda C$ is invertible for $0<|\lambda| \leq$

Remark 5.7. With the notation of Theorems 5.5 and 5.6, in the case when $\Gamma_{\varepsilon}$ and $\Xi_{\varepsilon}$ are singletons for all $\varepsilon \in[-a, a]$, the multivalued maps $\varepsilon \mapsto \Gamma_{\varepsilon}$ and $\varepsilon \vdash \Xi_{\varepsilon}$ can be regarded as ordinary (single valued) maps. In this case, the upper semicontinuity coincides with the continuity.

Theorem 5.8 (Existence of bifurcation points). Let $L, C, N$ and $\Omega$ be as in (5.1). Assume that $\Sigma=\partial \Omega \cap \operatorname{Ker} L$ is nonempty, compact, and that $\operatorname{Ker} L$ is odd dimensional. Then, $\Sigma$ contains at least one bifurcation point of problem (5.1).

Proof. Given $c>0$, let $V_{c}=\{x \in E: \operatorname{dist}(x, \Sigma)\}$. Assume the assertion is false. Then, the compact set $\Sigma \times\{(0,0)\}$ of the trivial solutions of (5.1) admits a neighborhood $V_{c} \times \mathcal{O} \subseteq E \times \mathbb{R}^{2}$ in which any solution of (5.1) is trivial. Of course we may assume that $c>0$ is sufficiently small, so that Theorem 5.5 applies. Therefore, as a consequence of all the three assertions of Theorem 5.5, for any $\varepsilon \neq 0$ sufficiently small, $V_{c} \times \mathcal{O}$ contains a solution $(x, \varepsilon, \lambda)$ of $(5.1)$, which is nontrivial, being $\varepsilon \neq 0$. This contradiction implies our assertion.

Let $\gamma: E \rightarrow \mathbb{R}$ be a continuous norm on the Banach space $E$ and define the (possibly unbounded) open subset $\Omega=\{x \in E: \gamma(x)<1\}$ of $E$. Observe that, given any nontrivial finite dimensional subspace $E_{0}$ of $E$, the set $\partial \Omega \cap E_{0}$ is nonempty and compact. This is due to the fact that in a finite dimensional vector space all the norms are equivalent. Thus, Corollary 5.9 below is a straightforward consequence of Theorem 5.8. 
Corollary 5.9. Let $L, C: E \rightarrow F$ be as in (5.1) and let $N: E \rightarrow F$ be continuous and locally $\alpha$-Lipschitz. Assume that $\gamma: E \rightarrow \mathbb{R}$ is a continuous norm on $E$. If the dimension of Ker $L$ is odd, then the unit $\gamma$-sphere $\gamma^{-1}(1) \cap$ Ker $L$ of $\operatorname{Ker} L$ contains at least one bifurcation point of problem (5.1) in which $\Omega=\{x \in E: \gamma(x)<1\}$.

\section{EXAmples}

In this section we provide examples in which our results apply, as well as examples showing that the assumption that the (geometric) multiplicity of the eigenvalue $\lambda=0$ (for the unperturbed problem) is odd cannot be dropped.

The following is, if we may say so, a multi-example: it provides problems for which the multiplicity of the eigenvalue $\lambda_{0}=0$ is an arbitrary positive integer.

Example 6.1. Given $k \in \mathbb{N}$, let $L_{k}$ be the bounded linear operator in $\ell^{2}$ that to any $x=\left(\xi_{1}, \xi_{2}, \ldots\right) \in \ell^{2}$ associates the element

$$
L_{k} x=(\underbrace{0,0, \ldots, 0}_{k \text { entries }}, \xi_{k+1}, \xi_{k+2}, \ldots),
$$

and define the nonlinear map $N: \ell^{2} \rightarrow \ell^{2}$ by

$$
N(x)=\left(-\xi_{2}, \sigma\left(\xi_{1}\right),-\xi_{4}, \sigma\left(\xi_{3}\right), \ldots,-\xi_{2 i}, \sigma\left(\xi_{2 i-1}\right), \ldots\right),
$$

where $\sigma: \mathbb{R} \rightarrow \mathbb{R}$ is given by

$$
\sigma(t)=\frac{3 t+|t|}{2}=\left\{\begin{aligned}
t & \text { if } t<0 \\
2 t & \text { if } t \geq 0
\end{aligned}\right.
$$

Observe that $L_{k}$ is Fredholm of index zero and its kernel is the $k$-dimensional space

$$
\text { Ker } L_{k}=\left\{x \in \ell^{2}: x=\left(\xi_{1}, \xi_{2}, \ldots \xi_{k}, 0,0, \ldots\right)\right\} ;
$$

so that, $\lambda_{0}=0$ is an eigenvalue of $L_{k}$ of geometric multiplicity $k$.

Notice also that $N$ is a non-differentiable Lipschitz map and, consequently, it is $\alpha$-Lipschitz as well.

Consider the problem

$$
\left\{\begin{array}{l}
L_{k} x+\varepsilon N(x)=\lambda x \\
\gamma(x)=1
\end{array}\right.
$$

where $\gamma(x)=\|x\|_{\infty}$ is the $\ell^{\infty}$ norm on $\ell^{2}$, which is clearly continuous with the $\ell^{2}$ topology. The transversality condition (5.2) is satisfied since, in this case, the operator $C$ is the identity.

It is easy to verify that, if $k$ is even, then, for any $\varepsilon \neq 0$ and for any $\lambda$, the equation

$$
L_{k} x+\varepsilon N(x)=\lambda x
$$

admits only the trivial solution. On the other hand, if $k$ is odd, according to Corollary 5.9, problem (6.1) admits at least one bifurcation point. 
For example, if $k=3$, then for any sufficiently small $\varepsilon \neq 0$ we get just two "small" $\partial \Omega$-eigenvalues, where $\Omega$ is the unit $\gamma$-ball in $E$,

$\lambda_{\varepsilon}^{-}=\frac{1-\sqrt{1-4 \varepsilon^{2}}}{2}=\varepsilon^{2}+\mathrm{o}\left(\varepsilon^{2}\right) \quad$ and $\quad \lambda_{\varepsilon}^{+}=\frac{1-\sqrt{1-8 \varepsilon^{2}}}{2}=2 \varepsilon^{2}+\mathrm{o}\left(\varepsilon^{2}\right)$, of $L_{3}+\varepsilon N$ to which correspond, respectively, the $\partial \Omega$-eigenvectors

$$
v_{\varepsilon}^{-}=\left(0,0,-1, \xi_{4}^{-}(\varepsilon), 0, \ldots\right) \quad \text { and } \quad v_{\varepsilon}^{+}=\left(0,0,1, \xi_{4}^{+}(\varepsilon), 0, \ldots\right),
$$

where

$$
\xi_{4}^{-}(\varepsilon)=\frac{\sqrt{1-4 \varepsilon^{2}}-1}{2 \varepsilon}=-\varepsilon+\mathrm{o}(\varepsilon)
$$

and

$$
\xi_{4}^{+}(\varepsilon)=\frac{\sqrt{1-8 \varepsilon^{2}}-1}{2 \varepsilon}=-2 \varepsilon+\mathrm{o}(\varepsilon) .
$$

Thus, we obtain exactly two bifurcation points for the perturbed eigenvalue problem

$$
\left\{\begin{array}{l}
L_{3} x+\varepsilon N(x)=\lambda x \\
\gamma(x)=1
\end{array}\right.
$$

namely $x_{0}^{-}=(0,0,-1,0, \ldots)$ and $x_{0}^{+}=(0,0,1,0, \ldots)$.

In the following example, whose physical meaning is evident, $\lambda_{0}=0$ is an eigenvalue of multiplicity 2 of the unperturbed problem. Although the transversality condition (5.2) is satisfied, the persistence of the eigenvalues of the perturbed problem does not subsist.

Example 6.2. Consider the problem

$$
\left\{\begin{array}{l}
x^{\prime \prime}+x+\varepsilon x^{\prime}=\lambda x \\
x(0)=x(2 \pi), x^{\prime}(0)=x^{\prime}(2 \pi),
\end{array}\right.
$$

and observe that, when $\varepsilon \neq 0$, whatever is $\lambda$, it admits only the trivial solution.

Here, in this example, let $E$ denote the 2-codimensional closed subspace of the Banach space $C^{2}([0,2 \pi], \mathbb{R})$ of those functions $x$ satisfying the $2 \pi$ periodic boundary conditions $x(0)=x(2 \pi)$ and $x^{\prime}(0)=x^{\prime}(2 \pi)$, and put $F=C([0,2 \pi], \mathbb{R})$. System $(6.2)$ can be written in the form

$$
L x+\varepsilon N(x)=\lambda C x,
$$

where

- $L: E \rightarrow F$ is the operator $x \mapsto x^{\prime \prime}+x$;

- $N: E \rightarrow F$ is the (linear) map $x \mapsto x^{\prime}$;

- $C$ is the inclusion of $E$ into $F$.

Notice that $L$ is a Fredholm operator of index zero, as the composition of the inclusion $E \hookrightarrow C^{2}([0,2 \pi], \mathbb{R})$, which is Fredholm of index -2 , with the operator $x \in C^{2}([0,2 \pi], \mathbb{R}) \mapsto x^{\prime \prime}+x \in F$, which is Fredholm of index 2 (being surjective with 2-dimensional kernel). The kernel of $L$ is spanned by $\sin t$ and $\cos t$. Therefore, the transversality condition (5.2) is satisfied 
since these two independent elements of $\operatorname{Ker} L$ do not belong to the image of $L$ (which has codimension 2 in $F$ ). The map $N$ is clearly $\alpha$-Lipschitz, being a bounded linear operator. Actually, because of Ascoli's theorem, $N$ is a compact operator.

Since, as already observed, when $\varepsilon \neq 0$, problem (6.2) admits only the trivial solution, given any open set $\Omega$ containing the origin, the persistence of $\partial \Omega$-eigenvectors does not subsist. This shows that, in Theorems 5.5 and 5.6 , as well as in Theorem 5.8, the assumption that Ker $L$ is odd dimensional cannot be removed.

The following is an example of a system of two coupled second order differential equations in which condition (5.2) is satisfied and the eigenvalue of the unperturbed problem has multiplicity 3. Consequently, the persistence phenomenon occurs.

Example 6.3. Consider the following system of coupled differential equations with $2 \pi$-periodic boundary conditions:

$$
\left\{\begin{array}{l}
x^{\prime \prime}+x+\varepsilon \cos t=\lambda x \\
y^{\prime \prime}-x^{\prime \prime}+\varepsilon\left|x^{\prime \prime}\right|=\lambda y \\
x(0)=x(2 \pi), x^{\prime}(0)=x^{\prime}(2 \pi), \\
y(0)=y(2 \pi), y^{\prime}(0)=y^{\prime}(2 \pi) .
\end{array}\right.
$$

Notice that the solutions $(x, y)$ of $(6.3)$ are all of class $C^{2}$ (actually $x \in$ $\left.C^{\infty}\right)$. Thus, we may seek for them in the subspace $E$ of the Banach space $C^{2}\left([0,2 \pi], \mathbb{R}^{2}\right)$ of those pairs $(x, y)$ satisfying the $2 \pi$-periodic boundary conditions of system (6.3). Notice that $E$ has codimension 4 in $C^{2}\left([0,2 \pi], \mathbb{R}^{2}\right)$ and, consequently, the operator

$$
L: E \rightarrow F=C\left([0,2 \pi], \mathbb{R}^{2}\right) \text { given by }(x, y) \mapsto\left(x^{\prime \prime}+x, y^{\prime \prime}-x^{\prime \prime}\right)
$$

is Fredholm of index zero. Elementary computations show that the unperturbed problem has $\lambda_{0}=0$ as an eigenvalue whose eigenspace $\operatorname{Ker} L$ is 3 -dimensional and is spanned by the following three pairs of functions:

$$
(\sin t, \sin t), \quad(\cos t, \cos t), \quad(0,1) .
$$

Consider, in $E$, the continuous norm

$$
\gamma((x, y))=\sup \{|x(t)|+|y(t)|: t \in[0,2 \pi]\} .
$$

Observe that the non-differentiable map $N: E \rightarrow F$, given by

$$
N(x)(t)=\left(\cos t,\left|x^{\prime \prime}(t)\right|\right),
$$

is Lipschitz and, consequently, $\alpha$-Lipschitz.

As we will see, the transversality condition (5.2) is satisfied with $C$ the inclusion of $E$ into $F$. Thus, according to Corollary 5.9, problem (6.3) together with the condition $\gamma(x, y)=1$ admits some bifurcation points. Let us see if we can find them.

When $\lambda=0$, the first equation $x^{\prime \prime}+x+\varepsilon \cos t=\lambda x$ has no $2 \pi$-periodic solutions, except in the case when $\varepsilon=0$, which is of no interest for our 
search for bifurcation points. Therefore, we may suppose $\lambda \neq 0$ and, of course, small. In this case the first equation admits only one $2 \pi$-periodic solution:

$$
x_{(\varepsilon, \lambda)}(t)=\frac{\varepsilon}{\lambda} \cos t .
$$

Replacing this solution in the second differential equation we get

$$
y^{\prime \prime}-\lambda y=-\frac{\varepsilon}{\lambda} \cos t-\varepsilon\left|\frac{\varepsilon}{\lambda} \cos t\right|,
$$

which, for small $\lambda \neq 0$, admits a unique $2 \pi$-periodic solution, say $y_{(\varepsilon, \lambda)}(t)$. Thus

$$
y_{(\varepsilon, \lambda)}(t)=\frac{\varepsilon}{\lambda(1+\lambda)} \cos t+\frac{\varepsilon}{\lambda}\left|\frac{\varepsilon}{\lambda}\right| u_{\lambda}(t),
$$

where $u_{\lambda}(t)$ is the unique $2 \pi$-periodic solution (for small $\lambda \neq 0$ ) of

$$
y^{\prime \prime}=\lambda(y-|\cos t|) \text {. }
$$

Let $C_{2 \pi}^{2}([0,2 \pi], \mathbb{R})$ denote the Banach space of the $C^{2}$ functions

$$
y:[0,2 \pi] \rightarrow \mathbb{R}
$$

satisfying the $2 \pi$-periodic conditions $y(0)=y(2 \pi), y^{\prime}(0)=y^{\prime}(2 \pi)$. If, for $\lambda \rightarrow 0, u_{\lambda}$ admits a limit in $C_{2 \pi}^{2}([0,2 \pi], \mathbb{R})$, then this limit must be a constant function (i.e. a $2 \pi$-periodic solution of the limit equation $y^{\prime \prime}=0$ ), and the constant must be $2 / \pi$, which is the average in the interval $[0,2 \pi]$ of $|\cos t|$. In fact, by integrating the last equation from 0 to $2 \pi$, one gets the identity

$$
\int_{0}^{2 \pi}\left(u_{\lambda}(t)-|\cos t|\right) d t=0, \quad \text { for small } \lambda \neq 0,
$$

which, passing to the limit for $\lambda \rightarrow 0$, implies the assertion.

One can check that the existence of such a limit is ensured by the celebrated Crandall-Rabinowitz Theorem [16]. We sketch the idea of how to see this. The details are left to the reader.

Observe first that

$$
f: \mathbb{R} \times C_{2 \pi}^{2}([0,2 \pi], \mathbb{R}) \rightarrow C([0,2 \pi], \mathbb{R}), \quad(\lambda, y) \mapsto y^{\prime \prime}-\lambda(y-\cos (\cdot))
$$

is a $C^{2}$ (actually $C^{\infty}$ ) Fredholm map of index one. Then regard the line

$$
\mathcal{L}=\left\{\left(0, c^{-}\right): c \in \mathbb{R}, c^{-} \text {the constant } c \text {-valued function defined on }[0,2 \pi]\right\}
$$

as the 1-dimensional manifold of trivial solutions of the equation $f(\lambda, y)=0$, so that the notion of bifurcation point makes sense: it is an element of $\mathcal{L}$ which is an accumulation point of nontrivial solutions.

Now check that

$$
p=\left(0, m_{0}^{-}\right) \in \mathcal{L},
$$

where $m_{0}=2 / \pi$, is the only element in the set of trivial solutions satisfying the necessary condition for bifurcation: the kernel of the derivative of $f$ at $p, f^{\prime}(p)$, has dimension bigger than 1 (i.e. it contains properly the tangent space of $\mathcal{L}$ at $p)$.

After this, observe that the second derivative of $f$ at any point $(\lambda, y)$, $f^{\prime \prime}(\lambda, y)$, is the bilinear symmetric operator given by $\left(\left(\mu_{1}, u_{1}\right),\left(\mu_{2}, u_{2}\right)\right) \mapsto$ 
$\mu_{1} u_{2}+\mu_{2} u_{1}$. Incidentally, we observe that this derivative does not depend on the point in which it is computed (therefore, the third derivative of $f$ is zero, and $f$ is $C^{\infty}$ ).

Finally, check that the second derivative of $f$ at $p$, regarded as a bilinear operator from the (2-dimensional) kernel to the (1-dimensional) cokernel of $f^{\prime}(p)$, satisfies the Crandall-Rabinowitz sufficient condition for the set of solutions of $f(\lambda, y)=0$ to admit a neighborhood of $p$ diffeomorphic to $\left\{(r, s) \in \mathbb{R}^{2}: r s=0\right\}$.

Put $m_{\lambda}=\sup _{t \in[0,2 \pi]}\left|u_{\lambda}(t)\right|$ and observe that

$$
\gamma\left(\left(x_{(\varepsilon, \lambda)}, y_{(\varepsilon, \lambda)}\right)\right)=\left|\frac{\varepsilon}{\lambda}\right|\left(1+\frac{1}{1+\lambda}+\left|\frac{\varepsilon}{\lambda}\right| m_{\lambda}\right) .
$$

Thus, for $\varepsilon$ and $\lambda$ small, the solution $\left(x_{(\varepsilon, \lambda)}, y_{(\varepsilon, \lambda)}\right)$ of system (6.3) belongs to the unit $\gamma$-sphere of $E$ if and only if

$$
1=\nu\left(1+\frac{1}{1+\lambda}+\nu m_{\lambda}\right) \approx m_{0} \nu^{2}+2 \nu
$$

where $\nu=|\varepsilon / \lambda|$ and $m_{0}=2 / \pi=\lim _{\lambda \rightarrow 0} m_{\lambda}$. Hence, recalling that $\nu>0$, we get

$$
\nu=\frac{-1+\sqrt{1+m_{0}}}{m_{0}} \approx 0.43873
$$

Consequently, the unit (2-dimensional) $\gamma$-sphere of $\operatorname{Ker} L$ has exactly two (antipodal) bifurcation points:

$$
\pm \nu\left(\cos t, \cos t+m_{0} \nu\right) \approx \pm 0.43873(\cos t, \cos t+0.27930)
$$

Finally, as promised, let us check that condition (5.2) is satisfied. To this purpose, we will show that the following equivalent condition (see (5.4) in Remark 5.1) holds true:

$$
(u \in \operatorname{Ker} L) \wedge(C u \in \operatorname{Im} L) \Longrightarrow u=0 .
$$

Let $u$ be a generic element in Ker $L$. That is,

$$
u(t)=a(\sin t, \sin t)+b(\cos t, \cos t)+c(0,1), \quad a, b, c \in \mathbb{R} .
$$

We need to show that, if the system

$$
\left\{\begin{array}{l}
x^{\prime \prime}+x=a \sin t+b \cos t \\
y^{\prime \prime}-x^{\prime \prime}=a \sin t+b \cos t+c
\end{array}\right.
$$

has at least one $2 \pi$-periodic solution, then $a=b=c=0$.

The first equation has no $2 \pi$-periodic solutions, unless $a=b=0$, and in this case has many of them, all of the type $x(t)=\alpha \sin t+\beta \cos t$. Replacing any one of these solutions in the second equation, we get

$$
y^{\prime \prime}=c-\alpha \sin t-\beta \cos t .
$$

But this equation has a $2 \pi$-periodic solution (if and) only if the average, in the interval $[0,2 \pi]$, of its second member is zero, which is true (if and) only if $c=0$. 
We conclude with an example in which the perturbing operator is continuous but not Lipschitz continuous (as in [11]).

Example 6.4. Consider the problem

$$
\left\{\begin{array}{l}
x^{\prime \prime}+x^{\prime}+\varepsilon t \sqrt{|x|}=\lambda x, \\
x^{\prime}(0)=x^{\prime}(1)=0, \\
\gamma(x)=1
\end{array}\right.
$$

where $\gamma(x)=\sup \{|x(t)|: t \in[0,1]\}$.

Here, let $E$ denote the 2-codimensional closed subspace of the Banach space $C^{2}([0,1], \mathbb{R})$ of the functions $x$ satisfying the boundary conditions $x^{\prime}(0)=0$ and $x^{\prime}(1)=0$, and put $F=C([0,1], \mathbb{R})$. The above problem can be written in the form

$$
\left\{\begin{array}{l}
L x+\varepsilon N(x)=\lambda C x \\
x \in \partial \Omega
\end{array}\right.
$$

where

- $L: E \rightarrow F$ is the bounded linear operator $x \mapsto x^{\prime \prime}+x^{\prime}$;

- $N: E \rightarrow F$ is the (non-differentiable) map defined by $N(x)(t)=$ $t \sqrt{|x(t)|}$

- $C$ is the inclusion of $E$ into $F$;

- $\Omega=\{x \in E: \gamma(x)<1\}$.

The operator $L$ is Fredholm of index zero, as the composition of the inclusion $E \hookrightarrow C^{2}([0,1], \mathbb{R})$, which is Fredholm of index -2 , with the differential operator $x \in C^{2}([0,1], \mathbb{R}) \mapsto x^{\prime \prime}+x^{\prime} \in F$, which is Fredholm of index 2 .

The kernel of $L$ is 1-dimensional and consists of the constant functions. Therefore, the transversality condition (5.2) is satisfied since the image of $L$ (made up of functions with zero average) does not contain the kernel of $L$ (regarded in $F$ ).

The map $N$ is $\alpha$-Lipschitz. Actually, it is completely continuous, due to the compact inclusion of $E$ into $F$.

The set $\partial \Omega \cap \operatorname{Ker} L$ is compact, since $\gamma$ is a norm in $E$ and $\operatorname{Ker} L$ is finite dimensional.

Since all the assumptions of Theorems 5.5 and 5.6 are satisfied, for any $\varepsilon$ sufficiently small, it should be possible to find $\lambda$ small and $x \in \partial \Omega$ close to Ker $L$ such that

$$
L x+\varepsilon N(x)=\lambda C x .
$$

In fact, numerically, for $\varepsilon=-0.1,0.1$ and 0.2 we have found the following values of the corresponding eigenvalue $\lambda$, as well as the initial and final conditions of the corresponding eigenfunction $x \in \partial \Omega$ (so that the reader can verify):

$(-0.1) \lambda \approx-0.05830605, x(0)=1, x^{\prime}(0)=0, x(1) \approx 0.99179393,\left|x^{\prime}(1)\right|<$ $10^{-8}$; 
$(+0.1) \lambda \approx 0.05832793, x(0) \approx 0.99184418,\left|x^{\prime}(0)\right|<10^{-8}, x(1)=1$, $x^{\prime}(1)=0$

$(+0.2) \lambda \approx 0.11691529, x(0) \approx 0.98377161,\left|x^{\prime}(0)\right|<10^{-8}, x(1)=1$, $x^{\prime}(1)=0$.

\section{REFERENCES}

[1] Appell J. - De Pascale E. - Vignoli A., Nonlinear spectral theory, de Gruyter, Berlin, 2004.

[2] Benevieri P. - Calamai A. - Furi M., A degree theory for a class of perturbed Fredholm maps, Fixed Point Theory Appl. 2 (2005), 185-206.

[3] Benevieri P. - Calamai A. - Furi M., A degree theory for a class of perturbed Fredholm maps II, Fixed Point Theory Appl. (2006), Art. ID 27154, 20 pp.

[4] Benevieri P. - Calamai A. - Furi M., On the degree for oriented quasi-Fredholm maps: its uniqueness and its effective extension of the Leray-Schauder degree, Topol. Methods in Nonlin. Anal. 46 (2015), 401-430.

[5] Benevieri P. - Furi M., A simple notion of orientability for Fredholm maps of index zero between Banach manifolds and degree theory, Ann. Sci. Math. Québec 22 (1998), 131-148.

[6] Benevieri P. - Furi M., On the concept of orientability for Fredholm maps between real Banach manifolds, Topol. Methods Nonlinear Anal. 16 (2000), 279-306.

[7] Benevieri P. - Furi M., Bifurcation results for families of Fredholm maps of index zero between Banach spaces, Nonlinear analysis and its applications (St. John's, NF, 1999), Nonlinear Analysis Forum 6 (2001), 35-47.

[8] Benevieri P. - Furi M., A Degree for locally compact perturbations of Fredholm maps in Banach spaces, Abstract and Applied Analysis, 2006, Art. ID 64764, 20 pp.

[9] Benevieri P. - Furi M. - Pera M.P. - Spadini M., About the sign of oriented Fredholm operators between Banach spaces, Z. Anal. Anwendungen 22 (2003), no. 3, 619-645.

[10] Calamai A., The Invariance of Domain Theorem for compact perturbations of nonlinear Fredholm maps of index zero, Nonlinear Funct. Anal. Appl. 9 (2004), 185-194.

[11] Chiappinelli R., Isolated Connected Eigenvalues in Nonlinear Spectral Theory, Nonlinear Funct. Anal. Appl. 8 (2003), 557-579.

[12] Chiappinelli R. - Furi M. - Pera M.P., Normalized Eigenvectors of a Perturbed Linear Operator via General Bifurcation, Glasgow Mathematical Journal 50 (2008), 303318.

[13] Chiappinelli R. - Furi M. - Pera M.P., Topological persistence of the normalized eigenvectors of a perturbed self-adjoint operator, Applied Mathematics Letters 23 (2010), 193-197.

[14] Chiappinelli R. - Furi M. - Pera M.P., Persistence of the normalized eigenvectors of a perturbed operator in the variational case, Glasg. Math. J. 55 (2013), no. 3, 629-638.

[15] Chiappinelli R. - Furi M. - Pera M.P., Topological Persistence of the Unit Eigenvectors of a Perturbed Fredholm Operator of Index Zero, Z. Anal. Anwend. 33 (2014), 347367.

[16] Crandall M.G. - Rabinowitz P.H., Bifurcation from simple eigenvalues, J. Funct. Anal. 8 (1971), 321-340.

[17] Darbo G., Punti uniti in trasformazioni a codominio non compatto, Rend. Sem. Mat. Univ. Padova 24 (1955), 84-92.

[18] Deimling K., Nonlinear Functional Analysis, Springer, Berlin, 1985.

[19] Edmunds D.E. - Webb J.R.L., Remarks on nonlinear spectral theory, Boll. Un. Mat. Ital. B (6) 2 (1983), 377-390.

[20] Furi M. - Martelli M. - Vignoli A., Contributions to spectral theory for nonlinear operators in Banach spaces, Ann. Mat. Pura Appl. 118 (1978), 229-294. 
[21] Kato T., Perturbation theory for linear operators, Classics in Mathematics, SpringerVerlag, Berlin, 1995.

[22] Kuratowski C., Topologie, Monografie Matematyczne, 20, Warszawa, 1958.

[23] Pejsachowicz J. - Rabier P., Degree theory for $C^{1}$ Fredholm mappings, J. Anal. Math. 76 (1998), 289-319.

[24] Rabinowitz P.H., Some global results for nonlinear eigenvalue problems, J. Funct. Anal. 7 (1971), 487-513.

[25] Smale S., An infinite dimensional version of Sard's theorem, Amer. J. Math. 87 (1965), 861-866.

[26] Taylor A.E. - Lay D.C., Introduction to Functional Analysis, John Wiley \& Sons, New York-Chichester-Brisbane, 1980.

[27] Väth M., Topological Analysis: From the Basics to the Triple Degree for Nonlinear Fredholm Inclusions, de Gruyter, Berlin, New York, 2012.

Pierluigi Benevieri - Dipartimento di Matematica e Informatica, Università degli Studi di Firenze, Via S. Marta 3, I-50139 Firenze, Italy and Instituto de Matemática e Estatística, Universidade de São Paulo, Rua do Matão 1010, São Paulo - SP - Brasil CEP 05508-090 - E-mail address: pierluigi.benevieri@unifi.it

Alessandro Calamai - Dipartimento di Ingegneria Industriale e Scienze Matematiche, Università Politecnica delle Marche, Via Brecce Bianche, I-60131 Ancona, Italy - Email address: calamai@dipmat.univpm.it

Massimo Furi - Dipartimento di Matematica e Informatica "Ulisse Dini", UniverSità degli Studi di Firenze, Via S. Marta 3, I-50139 Florence, Italy - E-mail address: massimo.furi@unifi.it

Maria Patrizia Pera - Dipartimento di Matematica e Informatica "Ulisse Dini", Università degli Studi di Firenze, Via S. Marta 3, I-50139 Florence, Italy - E-mail address: mpatrizia.pera@unifi.it 